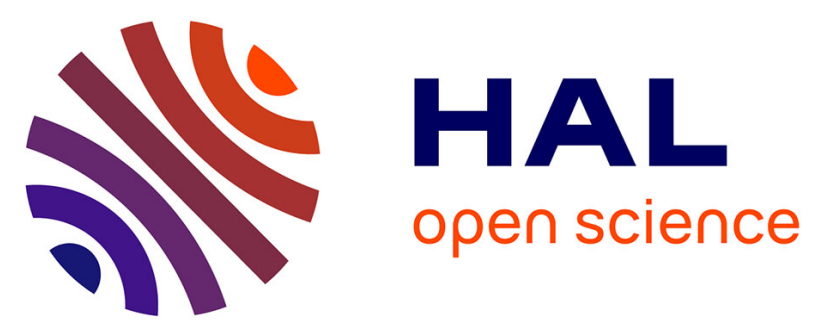

\title{
Healthy Life-Year Costs of Treatment Speed From Arrival to Endovascular Thrombectomy in Patients With Ischemic Stroke
}

Mohammed A Almekhlafi, Mayank Goyal, Diederik W J Dippel, Charles B L M Majoie, Bruce C V Campbell, Keith W Muir, Andrew M Demchuk, Serge

Bracard, Francis Guillemin, Tudor G Jovin, et al.

\section{To cite this version:}

Mohammed A Almekhlafi, Mayank Goyal, Diederik W J Dippel, Charles B L M Majoie, Bruce C V Campbell, et al.. Healthy Life-Year Costs of Treatment Speed From Arrival to Endovascular Thrombectomy in Patients With Ischemic Stroke. JAMA neurology, 2021, e211055, 10.1001/jamaneurol.2021.1055 . hal-03220460

\section{HAL Id: hal-03220460 \\ https://hal.univ-lorraine.fr/hal-03220460}

Submitted on 7 May 2021

HAL is a multi-disciplinary open access archive for the deposit and dissemination of scientific research documents, whether they are published or not. The documents may come from teaching and research institutions in France or abroad, or from public or private research centers.
L'archive ouverte pluridisciplinaire HAL, est destinée au dépôt et à la diffusion de documents scientifiques de niveau recherche, publiés ou non, émanant des établissements d'enseignement et de recherche français ou étrangers, des laboratoires publics ou privés. 


\title{
Healthy Life-Year Costs of Treatment Speed From Arrival to Endovascular Thrombectomy in Patients With Ischemic Stroke A Meta-analysis of Individual Patient Data From 7 Randomized Clinical Trials
}

\author{
Mohammed A. Almekhlafi, MD, MSc; Mayank Goyal, MD, PhD; Diederik W. J. Dippel, MD; Charles B. L. M. Majoie, MD, PhD; Bruce C. V. Campbell, MD; \\ Keith W. Muir, MD; Andrew M. Demchuk, MD; Serge Bracard, MD; Francis Guillemin, MD, PhD; Tudor G. Jovin, MD; Peter Mitchell, MD; \\ Philip White, MD; Michael D. Hill, MD, MSc; Scott Brown, PhD; Jeffrey L. Saver, MD; for the HERMES Trialists Collaboration
}

IMPORTANCE The benefits of endovascular thrombectomy (EVT) are time dependent. Prior studies may have underestimated the time-benefit association because time of onset is imprecisely known.

OBJECTIVE To assess the lifetime outcomes associated with speed of endovascular thrombectomy in patients with acute ischemic stroke due to large-vessel occlusion (LVO).

DATA SOURCES PubMed was searched for randomized clinical trials of stent retriever thrombectomy devices vs medical therapy in patients with anterior circulation LVO within 12 hours of last known well time, and for which a peer-reviewed, complete primary results article was published by August 1, 2020.

STUDY SELECTION All randomized clinical trials of stent retriever thrombectomy devices vs medical therapy in patients with anterior circulation LVO within 12 hours of last known well time were included.

DATA EXTRACTION/SYNTHESIS Patient-level data regarding presenting clinical and imaging features and functional outcomes were pooled from the 7 retrieved randomized clinical trials of stent retriever thrombectomy devices (entirely or predominantly) vs medical therapy. All 7 identified trials published in a peer-reviewed journal (by August 1, 2020) contributed data. Detailed time metrics were collected including last known well-to-door (LKWTD) time; last known well/onset-to-puncture (LKWTP) time; last known well-to-reperfusion (LKWR) time; door-to-puncture (DTP) time; and door-to-reperfusion (DTR) time.

MAIN OUTCOMES AND MEASURES Change in healthy life-years measured as disability-adjusted life-years (DALYs). DALYs were calculated as the sum of years of life lost (YLL) owing to premature mortality and years of healthy life lost because of disability (YLD). Disability weights were assigned using the utility-weighted modified Rankin Scale. Age-specific life expectancies without stroke were calculated from 2017 US National Vital Statistics.

RESULTS Among the 781 EVT-treated patients, 406 (52.0\%) were early-treated (LKWTP $\leq 4$ hours) and 375 (48.0\%) were late-treated (LKWTP $>4-12$ hours). In early-treated patients, LKWTD was 188 minutes (interquartile range, 151.3-214.8 minutes) and DTP 105 minutes (interquartile range, 76-135 minutes). Among the 298 of 380 (78.4\%) patients with substantial reperfusion, median DTR time was 145.0 minutes (interquartile range, 111.5-185.5 minutes). Care process delays were associated with worse clinical outcomes in LKW-to-intervention intervals in early-treated patients and in door-to-intervention intervals in early-treated and late-treated patients, and not associated with LKWTD intervals, eg, in early-treated patients, for each 10-minute delay, healthy life-years lost were DTP 1.8 months vs LKWTD 0.0 months; $P<.001$. Considering granular time increments, the amount of healthy life-time lost associated with each 1 second of delay was DTP 2.2 hours and DTR 2.4 hours.

CONCLUSIONS AND RELEVANCE In this study, care delays were associated with loss of healthy life-years in patients with acute ischemic stroke treated with EVT, particularly in the postarrival time period. The finding that every 1 second of delay was associated with loss of 2.2 hours of healthy life may encourage continuous quality improvement in door-to-treatment times.

JAMA Neurol. doi:10.1001/jamaneurol.2021.1055

Published online May 3, 2021.
Supplemental content

Author Affiliations: Author affiliations are listed at the end of this article.

Group Information: A complete list of the members of HERMES Trialists Collaboration appears in the Supplement.

Corresponding Author: Mohammed A. Almekhlafi, MD, MSc, Department of Clinical Neurosciences, University of Calgary, 1403 29th St NW, Calgary, AB T2N 2T9, Canada (mohammed. almekhlafi1@ucalgary.ca). 
$\mathrm{E}$ ndovascular thrombectomy (EVT) has produced substantial changes in the outcomes associated with acute ischemic stroke due to large-vessel occlusions. ${ }^{1}$ Evidence from multiple randomized clinical trials has demonstrated substantial EVT benefit, establishing thrombectomy as a standard of care treatment and encouraging efforts to improve its delivery. ${ }^{2}$ One of the most important determinants of outcome after acute ischemic stroke is rapid reperfusion via intravenous thrombolysis, EVT, or both, reducing total brain ischemia time. ${ }^{3-5}$

Previous analyses of the association of faster treatment delivery with improved patient outcomes have been limited. Ischemic stroke is a disease that can produce multiple gradations of functional impairment. For almost a decade, the preponderance of time-benefit studies focused on binary functional outcomes, counting only a single transition among disability states as important instead of all the transitions valued by patients, families, and practitioners. Even multilevel outcome analyses have generally analyzed disability level as an ordinal outcome, neglecting to weight outcome states using health utilities to capture health-related quality of life in a patient-centric manner.

Most analyses of EVT time-benefit associations have emphasized the time interval from onset/last known well (LKW) time to last known well/onset-to-puncture (LKWTP) time. ${ }^{6}$ The analyses of time-benefit associations using LKWTP time are imprecise and systematically biased toward the null. The imprecision arises because, even in patients with stroke starting while awake, the exact moment of symptom onset may have been incorrectly perceived or reported, especially in patients who were not with witnesses at time of onset, owing to strokeinduced deficits such as neglect or aphasia. A systematic bias toward the null occurs when patients with wake-up or unwitnessed-onset strokes are included in analyses. Among these patients, the last known well time precedes actual stroke start time, yielding artificially long LKWTP values and causing systematic underestimation of the association between speed of treatment delivery and amount of treatment benefit. In addition, the differential selection for EVT of only those latearriving patients who have limited extent of ischemic changes on imaging results in overrepresentation of patients with reduced speed of infarct growth also biasing toward undervaluing the time-benefit association.

To address these limitations, we undertook a series of analyses minimizing these sources of imprecision and bias toward the null, including by evaluating outcomes along the full health-related quality of life range, with lead analyses focused on onset-to-treatment times in patients arriving early, among whom patients with fast progression are likely to be fully represented, and door-to-treatment times, as their intervals begin with precisely known time of emergency department arrival rather than imprecisely known last known well time.

\section{Methods}

Study Design and Inclusion Criteria

We analyzed individual patient-level data included in the Highly Effective Reperfusion Evaluated in Multiple Endovas-

\section{Key Points}

Question What are the lifetime consequences associated with care process delays in patients with ischemic stroke who are treated with endovascular thrombectomy?

Findings In this meta-analysis of pooled individual patient data from 406 adults in 7 randomized trials, delays in delivering endovascular thrombectomy were associated with marked reductions in healthy life-years after treatment. In the time interval from hospital arrival to endovascular procedure start, every 1 second of delay was associated with loss of 2.2 hours of healthy life.

Meaning In this study, care delays in delivering endovascular thrombectomy to ischemic stroke patients were associated with substantial losses of healthy life-years; health care organization and workflow optimization should be a priority to facilitate faster reperfusion for acute stroke patients.

cular Stroke (HERMES) trials collaboration. The collaboration includes all randomized clinical trials of stent retriever thrombectomy devices (entirely or predominantly) vs medical therapy in patients with anterior circulation LVO within 12 hours of last known well time and for which a peerreviewed, complete primary results article was published by August 1, 2020. PubMed search and inquiry among collaborators and colleagues was performed to confirm that the 7 included represented all those eligible. ${ }^{7-13}$

These trials were approved by the respective local ethics boards and enrolled patients using prospective (6 trials) or prospective and deferred (1 trial) written informed consent. The details of the HERMES collaboration initiation and the method of the meta-analysis search strategy and data gathering have been previously reported. ${ }^{2,14}$ A full list of investigators in the HERMES collaboration is included in the Supplement.

Analyses were performed in 3 patient populations (all patients, early-treated patients, and late-treated patients). The early-treated population was defined as patients with an interval from last known well time to arterial puncture (LKWTP) of less than or equal to 4 hours. The 4-hour cutoff was selected based on spline analyses of both the HERMES clinical trial data set and the large Get With the GuidelineStroke national registry indicating that the first inflection point of change in time-benefit associations from linear to nonlinear occurs at an LKWTP of 240 minutes. ${ }^{4,5}$ This morphologic change was deemed to potentially indicate the transition point at which substantial numbers of unwitnessedonset patients, patients with symptoms first observed on awakening, and patients with fast progression begin to not be included in the EVT population.

The late-treated population was analyzed as an explanatory analysis, to probe a cohort likely to be more greatly biased by increased inclusion of unwitnessed onset and symptoms first observed on awakening and by a decreased inclusion of patients with fast progression. The late-treated population was defined as patients with LKWTP of greater than 4 hours and less than or equal to 12 hours.

All analyses were confined to directly arriving patients, excluding interfacility transfer patients. Transfer patients were 
identified in all trials and excluded because they often have paradoxically short emergency department to puncture (doorto-puncture [DTP]) times at the thrombectomy center, having undergone preliminary workup at outside facilities.

\section{Outcomes}

The leading outcome was gain or loss of healthy life, quantified using the disability-adjusted life-years (DALY) metric. The World Health Organization developed the DALY to measure the relative and total global burden of all diseases with a common metric. ${ }^{15}$ One DALY is 1 year of healthy life, free of disability. The DALY metric integrates both mortality (years of life lost [YLL] because of premature death) and functional outcome (years of healthy life lost because of living with disability [YLD]). DALYs were calculated as the sum of YLL plus YLD. DALY values for each patient were calculated based on their age at time of stroke, their global disability outcome level at 3 months after stroke, as measured by the modified Rankin Scale (mRS) (scale from 0 indicating no residual symptoms to 6 indicating death), and their resulting projected life expectance and health-related quality of life.

The YLL for each patient is the difference between the patient's age-specific life expectancy without stroke and the patient's age-specific life expectancy given the particular level of poststroke disability present at 3 months. Following current World Health Organization recommendations, age weighting and discount rates were not applied. Each patient's age-specific life expectancy without stroke was taken from 2017 US National Vital Statistics. The degree of life expectancy curtailment associated with poststroke mRS global disability level was based on 2 poststroke long-term cohort studies, the UK Lothian Cohort Study and the Swedish Risk-Stroke Cohort Study. ${ }^{16-18}$

The YLD for each patient was their years of expected life multiplied by a disability weight that reflected the degree of reduced value of each year owing to reduced health-related quality of life. Disability weights for each of the $7 \mathrm{mRS}$ global disability levels were assigned using the utility-weighted modified Rankin Scale (UW-mRS). For each mRS level, the disability weight was calculated as 1 minus the UW-mRS utility weight. The UW-mRS health-related quality of life weightings for each mRS level were derived by averaging findings from 2 studies, including a large population-based stroke patient study in Great Britain and an international stroke clinician study with US, European, and Asian participants. ${ }^{19,20}$ Accordingly, in the current study, for each mRS level, the disability weights were 0.0 for mRS level 0; 0.09 for mRS level 1; 0.24 for mRS level 2; 0.35 for mRS level 3; 0.67 for mRS level 4; 1.0 for mRS level 5; and 1.0 for mRS level $6 .^{21}$

An additional outcome analyzed was health-related quality of life exactly at 3 months after stroke, as reflected in 3-month UW-mRS scores. Utility measures are preference values that patients attach to their overall health status and integrate both positive and negative effects of an intervention into a single score between 0 (equal to death) and 1 (equal to perfect health).

Time Intervals

A total of 5 time intervals were analyzed: (1) LKW to door (LKWTD), (2) LKW to puncture (LKWTP), (3) LKW to reperfu- sion (LKWTR), (4) door to puncture (DTP), and (5) door to reperfusion (DTR). Substantial reperfusion was scored centrally by the HERMES collaboration central core laboratory and was defined as a score of $2 \mathrm{~b}-3$ (successful reperfusion) on the modified Thrombolysis in Cerebral Infarction (mTICI) scale (scores range from 0 indicating no perfusion to 3 indicating complete perfusion). Analyses of LKWTD, LKWTP, and DTP were performed in all patients meeting the entry LKWTP time criterion, regardless of whether substantial reperfusion was achieved. Analyses of LKWTR and DTR were performed in the subset of patients in whom substantial reperfusion was achieved.

\section{Statistical Analysis}

Probability of each outcome as a function of time was analyzed using mixed-method ordinal logistic regression, with trial and trial-by-treatment interaction as random-effects variables. In the main analyses, models were constructed of the linear dependence of the log odds of a particular outcome on time duration of care processes. Six patient feature variables were used as covariates in adjusted analyses: age (a linear variable), sex (binary variable), baseline stroke severity (National Institutes of Health Stroke Scale [NIHSS] score, with a range of 0-42; the higher the score, the greater the stroke severity, with 0 indicating no signs of stroke and 42 indicating coma), target occlusion location (a 3-level categorical variableinternal carotid artery [ICA], M1 middle cerebral artery [MCA], M2 MCA), entry Alberta Stroke Program Early Computed Tomography Score (ASPECTS; linear variable), and pretreatment intravenous alteplase; binary variable). Race/ethnicity was not included because collection of race data was legally prohibited in some countries where studies were performed and because race/ethnicity is not a known major independent factor associated with outcome from large-vessel ischemic stroke.

All effect size estimates are provided with their 95\% CIs. All $P$ values presented are 2 -sided, with values less than .05 indicating statistical significance, without adjustment for multiple comparisons. Statistical analyses were performed in SAS software, version 9.4 (SAS Institute Inc), and R, version 3.3 (R Foundation for Statistical Computing).

\section{Results}

Among the 871 patients allocated to EVT across the 7 trials, 781 patients (89.7\%) underwent EVT and 90 did not, most often because of reperfusion before EVT or tortuous vessels precluding navigation to the target occlusion. Among the 781 EVT-treated patients, 406 patients $(52.0 \%)$ were in the earlytreated (LKWTP $\leq 4$ hours) population and 375 patients (48.0\%) were within the late-treated (LKWTP $>4-12$ hours) population.

The presenting features of early-treated and late-treated EVT patients are shown in Table 1 . In the early-treated population, the mean (SD) age was 66.2 (13.4) years, 46.6\% were women, and the median pretreatment NIH Stroke Scale score was 18 (interquartile range [IQR], 14-21). Patients in the latetreated group were generally comparable, although substan- 


\begin{tabular}{|c|c|c|c|}
\hline \multirow[b]{2}{*}{ Characteristic } & \multicolumn{3}{|l|}{ No./total No. (\%) } \\
\hline & $\begin{array}{l}\text { All } \\
(n=781)\end{array}$ & $\begin{array}{l}\text { LKWTP } \leq 4 h \\
(n=406)\end{array}$ & $\begin{array}{l}\text { LKWTP >4 h } \\
(n=375)\end{array}$ \\
\hline \multicolumn{4}{|l|}{ Age, y } \\
\hline Mean (SD) [No.] & 65.5 (13.5) [781] & $66.2(13.4)[406]$ & 64.7 (13.5) [375] \\
\hline Median (IQR) & $67.0(57.0-76.0)$ & $68.0(58.0-76.9)$ & $66.0(55.0-75.5)$ \\
\hline Female sex & $367 / 781(47.0)$ & $189 / 406(46.6)$ & $177 / 375(47.2)$ \\
\hline Atrial fibrillation & $217 / 640(33.9)$ & $121 / 343(35.3)$ & $96 / 297(32.3)$ \\
\hline Hypertension & $426 / 779(54.7)$ & $238 / 405(58.8)$ & $188 / 374(50.3)$ \\
\hline Hyperlipidemia & $264 / 766(34.5)$ & $137 / 400(34.3)$ & $127 / 366(34.7)$ \\
\hline Diabetes & $120 / 780(15.4)$ & 69/405 (17.0) & $51 / 375(13.6)$ \\
\hline Prior stroke & $89 / 777(11.5)$ & 49/403 (12.2) & $40 / 374(10.7)$ \\
\hline \multicolumn{4}{|l|}{ Glucose level, mg/dL } \\
\hline Mean (SD) [No.] & 133.5 (79.6) [755] & $131.7(62.3)[394]$ & $135.5(95.1)$ [361] \\
\hline Median (IQR) & $120.0(106.2-140.4)$ & $118.8(105.6-140.0)$ & $121.0(107.3-141.8)$ \\
\hline \multicolumn{4}{|l|}{ Prestroke mRS } \\
\hline 0 & $501 / 605(82.8)$ & $255 / 314(81.2)$ & $246 / 291(84.5)$ \\
\hline 1 & $76 / 605(12.6)$ & $49 / 314(15.6)$ & $27 / 291(9.3)$ \\
\hline$\geq 2$ & $28 / 605(4.6)$ & $10 / 314(3.2)$ & $18 / 291(6.2)$ \\
\hline \multicolumn{4}{|l|}{ NIHSS at baseline } \\
\hline Mean (SD) [No.] & $17.0(4.8)[777]$ & $17.2(4.9)[403]$ & $16.9(4.7)[374]$ \\
\hline Median (IQR) & $17.0(14.0-21.0)$ & $18.0(14.0-21.0)$ & $17.0(14.0-20.0)$ \\
\hline tPA delivered & $678 / 781(86.8)$ & $366 / 406(90.1)$ & $312 / 375(83.2)$ \\
\hline \multicolumn{4}{|l|}{ Occlusion location } \\
\hline ICA & $198 / 733(27.0)$ & $103 / 387(26.6)$ & $95 / 346(27.5)$ \\
\hline $\begin{array}{l}\text { M1-segment middle } \\
\text { cerebral artery }\end{array}$ & $473 / 733(64.5)$ & $247 / 387(63.8)$ & $226 / 346(65.3)$ \\
\hline $\begin{array}{l}\text { M2-segment middle } \\
\text { cerebral artery }\end{array}$ & $61 / 733(8.3)$ & $37 / 387(9.6)$ & $24 / 346(6.9)$ \\
\hline Other & $1 / 733(0.1)$ & $0 / 387(0.0)$ & $1 / 346(0.3)$ \\
\hline \multicolumn{4}{|l|}{ ASPECTS at baseline } \\
\hline Mean (SD) [No.] & $7.6(1.8)$ [773] & $7.9(1.7)[401]$ & $7.3(1.9)$ [372] \\
\hline Median (IQR) & $8.0(7.0-9.0)$ & $8.0(7.0-9.0)$ & $7.0(6.0-9.0)$ \\
\hline $\mathrm{TICl} 2 \mathrm{~b} / 3$ & $536 / 728(73.6)$ & $298 / 380(78.4)$ & $238 / 348(68.4)$ \\
\hline \multicolumn{4}{|l|}{ LKW to randomization } \\
\hline Mean (SD) [No.] & $204.3(95.3)$ [779] & $144.6(37.9)[406]$ & $269.2(96.6)$ [373] \\
\hline Median (IQR) & $183.0(141.0-243.0)$ & $146.0(118.0-171.0)$ & $248.0(211.0-308.0)$ \\
\hline \multicolumn{4}{|l|}{ LKW to puncture } \\
\hline Mean (SD) [No.] & $249.4(94.0)[781]$ & $182.2(40.5)[406]$ & $322.3(80.3)[375]$ \\
\hline Median (IQR) & $240.0(185.0-299.0)$ & $188.0(151.3-214.8)$ & $300.0(270.0-346.5)$ \\
\hline \multicolumn{4}{|l|}{ LKW to reperfusion } \\
\hline Mean (SD) [No.] & $303.1(106.7)[520]$ & $233.9(63.7)[271]$ & 378.5 (92.1) [249] \\
\hline Median (IQR) & $291.5(230.8-358.3)$ & $234.0(190.5-274.0)$ & $358.0(315.0-410.0)$ \\
\hline \multicolumn{4}{|l|}{ ED to puncture } \\
\hline Mean (SD) [No.] & $121.2(57.0)[743]$ & $109.3(41.4)[376]$ & 133.5 (67.3) [368] \\
\hline Median (IQR) & $110.0(78.0-150.5)$ & $105.0(76.0-135.0)$ & $120.0(80.8-175.0)$ \\
\hline \multicolumn{4}{|l|}{ ED to reperfusion } \\
\hline Mean (SD) [No.] & $161.0(69.2)[383]$ & $152.9(57.6)[211]$ & $170.8(80.3)[172]$ \\
\hline Median (IQR) & $148.0(112.5-197.0)$ & $145.0(111.5-185.5)$ & $154.0(113.8-205.5)$ \\
\hline
\end{tabular}

Abbreviations: ASPECTS, Alberta Stroke Program Early Computed Tomography Score; ED, emergency department; ICA, internal carotid artery; IQR, interquartile range; LKW, last known well; LKWTP, last known well/onset to puncture; mRS, modified Rankin Scale (scale from 0 indicating no residual symptoms to 6 indicating death); NIHSS, National Institutes of Health Stroke Scale (with a range of 0-42; the higher the score, the greater the stroke severity, with 0 indicating no signs of stroke and 42 indicating coma); $\mathrm{TICl}$, thrombolysis in cerebral infarction (scores range from 0 indicating no perfusion to 3 indicating complete perfusion); tPA, tissue plasminogen activator.

SI conversion factor: To convert glucose to millimoles per liter, multiply by 0.0555 . tial reperfusion was achieved more often in early than in late patients (298 of 380 [78.4\%] vs 238 of 348 [68.4\%]).

Care process times in the patient treatment time window groups are shown in Table 1. In the early-treated population, the median LKWTD time was 188 minutes (IQR, 151.3-214.8 minutes) and median DTP time 105 minutes (IQR, 76-135 minutes). Among early-window patients in whom substantial reperfusion was achieved, median LKWTR time was 234 minutes (IQR, 190.5-274 minutes) and median DTR time 145 minutes (IQR, 111.5-185.5 minutes). 
Table 2. Outcomes Measured According to DALYs Lost and UW-mRS in the EVT Arm According to Various Epochs in the Early-Treatment or the Late-Treatment Population ${ }^{a}$

\begin{tabular}{|c|c|c|c|c|}
\hline \multirow[b]{2}{*}{ Time interval } & \multirow[b]{2}{*}{ Outcome } & \multicolumn{3}{|c|}{ EVT arm, effect per 1-h delay $(95 \% \mathrm{Cl})$} \\
\hline & & Full cohort & LKWTP $\leq 4 \mathrm{~h}$ & LKWTP >4 h \\
\hline \multirow[t]{2}{*}{ LKW to puncture } & UW-mRS & $-0.02(-0.04$ to -0.009$)$ & $-0.06(-0.11$ to -0.005$)$ & $-0.03(-0.06$ to -0.004$)$ \\
\hline & DALYc & $0.24(0.02$ to 0.46$)$ & 0.81 (0.08 to 1.55$)$ & $0.19(-0.22$ to 0.60$)$ \\
\hline \multirow[t]{2}{*}{ LKW to reperfusion } & UW-mRS & $-0.03(-0.05$ to -0.01$)$ & $-0.07(-0.11$ to -0.02$)$ & $-0.04(-0.07$ to -0.01$)$ \\
\hline & DALY & 0.33 (0.09 to 0.57$)$ & 0.84 (0.28 to 1.39$)$ & $0.50(0.07$ to 0.92$)$ \\
\hline \multirow[t]{2}{*}{ ED arrival to puncture } & UW-mRS & $-0.08(-0.11$ to -0.06$)$ & $-0.10(-0.15$ to -0.05$)$ & $-0.08(-0.11$ to -0.05$)$ \\
\hline & DALY & $0.93(0.56$ to 1.30$)$ & $0.92(0.16$ to 1.68$)$ & $0.86(0.43$ to 1.28$)$ \\
\hline \multirow[t]{2}{*}{ ED arrival to reperfusion } & UW-mRS & $-0.09(-0.12$ to -0.07$)$ & $-0.10(-0.15$ to -0.05$)$ & $-0.08(-0.11$ to -0.05$)$ \\
\hline & DALY & $1.00(0.67$ to 1.33$)$ & 0.84 (0.26 to 1.42$)$ & $1.00(0.60$ to 1.41$)$ \\
\hline \multirow[t]{2}{*}{ LKW to door } & UW-mRS & $0.002(-0.01$ to 0.02$)$ & $0.04(-0.01$ to 0.09$)$ & $0.02(-0.002$ to 0.04$)$ \\
\hline & DALY & $-0.04(-0.25$ to 0.16$)$ & $0.02(-0.69$ to 0.73$)$ & $-0.27(-0.57$ to 0.02$)$ \\
\hline \multicolumn{3}{|c|}{$\begin{array}{l}\text { Abbreviations: DALY, disability-adjusted life-year; ED, emergency department; } \\
\text { EVT, endovascular thrombosis; LKW, last known well; LKWTP, last known } \\
\text { well/onset to puncture; UW-mRS, utility-weighted modified Rankin Scale. }\end{array}$} & \multicolumn{2}{|c|}{$\begin{array}{l}\text { range in value from } 0 \text { (death) to } 1.00 \text { (optimum health). For example, in the } \\
\text { first row, the UW-mRS of }-0.02 \text { indicates that a patient's } 90 \text {-day UW-mRS will } \\
\text { be } 0.02 \text { points worse with every } 1 \text {-hour delay in LKW-to-puncture time. }\end{array}$} \\
\hline \multicolumn{3}{|c|}{$\begin{array}{l}\text { a The early-treatment population was defined as LKWTP } \leq 4 \text { hours and the } \\
\text { late-treatment population was defined as LKWTP }>4 \text { hours. }\end{array}$} & \multirow{2}{*}{\multicolumn{2}{|c|}{$\begin{array}{l}\text { ' For example, in the second row, the DALY of } 0.24 \text { indicates that a patient will } \\
\text { lose } 0.24 \text { years ( } 2.9 \text { months) of healthy life with every 1-hour delay in } \\
\text { LKW-to-puncture time. }\end{array}$}} \\
\hline \multicolumn{3}{|c|}{${ }^{\mathrm{b}}$ The UW-mRS is stated here in the conventional units of health utility, which } & & \\
\hline
\end{tabular}

The association of care process durations with functional outcomes is shown in Table 2 and the Figure. With regard to time intervals from LKW time to intervention, there was a difference between the early-treatment and late-treatment populations for the presence of a time-benefit association. In the early-treatment population, onset-to-puncture and onset-toreperfusion time intervals both showed substantial rates of benefit decrease with time, and the late-treatment population showed no significant change in benefit with longer onset-topuncture and onset-to-reperfusion time intervals. For example, in the early-treated population, every 1 hour of delay in LKW-to-puncture time was associated with a loss of 0.81 (95\% CI, 0.08-1.55) healthy life-years, and in the late-treated population no statistically significant association of LKW-topuncture time and DALYs was present. The early-treated population effect size is substantial. Considering more granular time intervals, the loss of 0.81 healthy years per hour delay in the early-treated patients is equivalent to a loss of 1.6 months per 10-minute delay, 4.9 days per 1-minute delay, and 2.0 hours per 1-second delay.

With regard to time intervals from ED arrival to intervention, the early-treatment and late-treatment populations both showed similar and substantial time-benefit associations for both door-to-puncture and door-to-reperfusion time intervals. For example, every 1 hour of delay in door-to-puncture was associated with a loss of 0.92 (95\% CI, 0.16-1.68) healthy life-years in the early-treated population and with a loss of 0.86 (95\% CI, 0.43-1.28) healthy life years in the late-treated population. Considering more granular time intervals, the loss of 0.92 healthy years per hour delay in early-treated patients is equivalent to a loss of 1.8 months per 10-minute delay, 5.6 days per 1-minute delay, and 2.2 hours per 1-second delay (Table 3).

In contrast to the time-benefit associations for door-tointervention time intervals, for the onset-to-door time interval, no statistically significant time-benefit association was pre- sent for either the early-treatment or the late-treatment population (Table 2).

\section{Discussion}

In this individual patient data pooled meta-analysis, substantial reductions in years of healthy life were associated in earlytreated patients with delays from last known well time to start of EVT procedures and to achievement of successful reperfusion and in both early-treated and late-treated patients with delays from door to procedure start and to reperfusion. Similar findings were noted for health-related quality of life at 3 months after stroke, as indexed by the UW-mRS. The timebenefit decrease was substantial: among early-treated patients, in the door-to-puncture time interval, each 1 hour of delay was associated with 0.92 healthy life-years lost and each 1 second of delay with 2.2 healthy life-hours lost.

In contrast, no association of treatment delay with outcomes was noted for several care process intervals that have less precision of temporal measurement, less representation of fast as well as slow progressors, or both. Of these, imprecision in recording timing of actual stroke onset likely is the greater factor. That time delays in the LKW-to-door interval were not associated with benefit decline in late-treated patients and in early-treated patients accords with the clinical recognition that times of stroke onset elicited by history are imprecise even in early-arriving patients who have reduced representation of wake-up and unwitnessed-onset strokes. Conversely, that time delays in the door-to-intervention interval were associated with benefit decrease in early-treated patients and in late-treated patients indicates that when time measures are fully reliable, as is the case with ED arrival, a substantial benefit decrease will be associated with time passage even fairly late after stroke onset. 
Figure. Association Between Postarrival Treatment Time and Disability-Adjusted Life-Years (DALYs) Lost After Endovascular Thrombectomy (EVT)

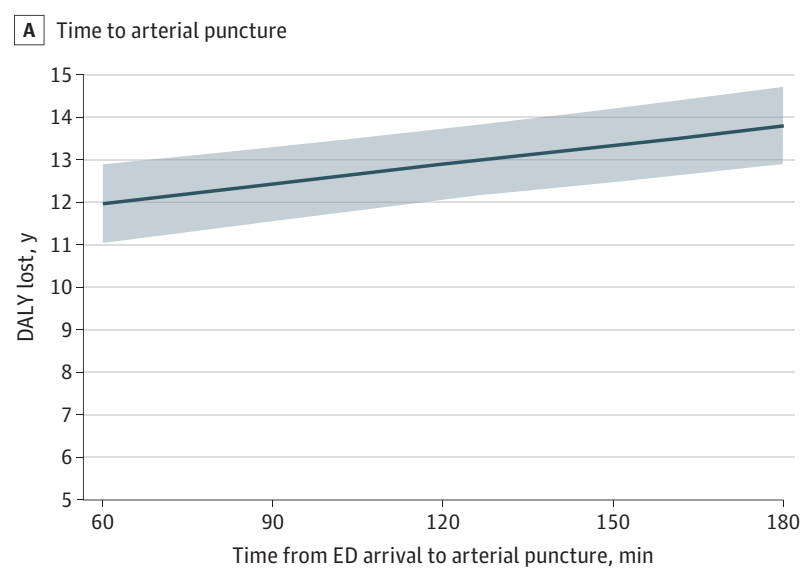

B Time to substantial perfusion

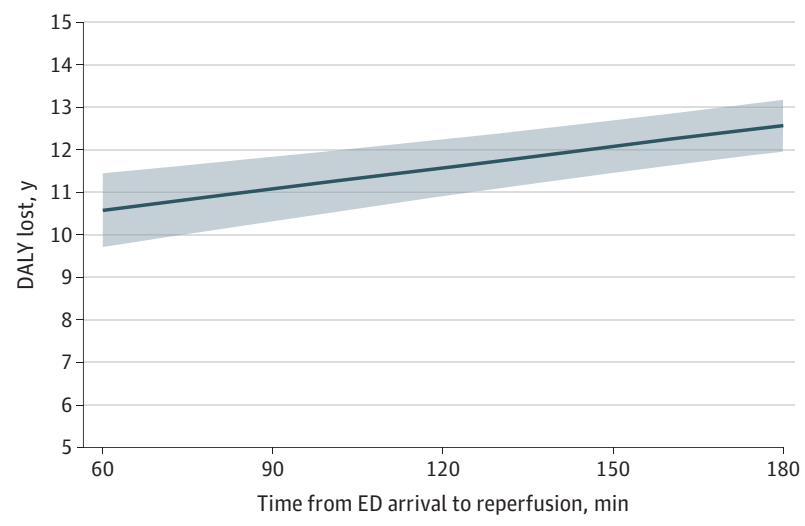

A, Years of healthy life lost by time from emergency department (ED) arrival to arterial puncture. B, Years of healthy life lost by time from ED arrival to substantial reperfusion. Curves were obtained from logistic regression of outcome on time as a continuous variable, after adjustment for age, sex, baseline stroke severity (National Institutes of Health Stroke Scale), target occlusion location, and concomitant intravenous tissue plasminogen activator. Solid curves indicate point estimates. Shaded areas indicate $95 \% \mathrm{Cls}$. Amount of healthy life-years lost is in relation to each individual patient's life expectancy had they not had a stroke based on 2017 US National Vital Statistics.

The association between EVT care delay and DALYs lost using an analysis confined to actual treatment times, actual patient ages, and actual outcomes among EVT-treated patients frames the analysis of the temporal dependency of benefit in unambiguous epochs of time. Prior studies estimated the effect of care delays on DALYs by applying patterns of mRS outcomes observed in clinical trials to an observational cohort not treated with $\mathrm{EVT}^{22}$ and a theoretical patient group with an imputed fixed rate of loss of eligibility for EVT throughout the first 6 hours after onset. ${ }^{6}$ Compared with these prior studies, the current investigation found moderately higher point estimates for the time-benefit decrease with treatment delay in EVT patients. The higher values may in part reflect the use of more reliably determined time points in the current study.
The current study is consonant with and extends prior studies that have reported much steeper decreases in benefit in dichotomous and ordinal mRS outcomes with postarrival care delays than with prearrival care days. ${ }^{4,5,23,24}$ This phenomenon has at least 3 potential sources. ${ }^{5}$ First is the application of imaging criteria after emergency department arrival to exclude patients who have already developed large core infarcts from endovascular treatment. Thus, imaging removes patients with wake-up onset and unwitnessed onset in whom symptoms' first observed time was long after actual stroke onset with interval advanced infarct progression. Passing through this process is a population to be treated with EVT, all of whom have clinical-core, perfusion-core, or collateral-core mismatch. The resulting selected patients are physiologically homogeneous, all having a propensity for rapid infarct growth in postarrival minutes. This phenomenon has been termed the time-reset effect by Fiehler. ${ }^{25}$ Second is the differential greater reliability of documented times in emergency department vs those for both unwitnessed and even witnessed stroke onset. Third is the possibility that human cerebral ischemic injury may follow an exponential or sigmoid growth trajectory, with more rapid progression at intermediate than very early aftersymptom onset times. The similarity of door-to-intervention time-benefit measures in early-arriving vs late-arriving patients suggests this third mechanism may be unlikely to be a major factor, as it would be expected to magnify early more than late associations.

The decline in EVT benefit with delay in door-topuncture and door-to-reperfusion time intervals in the current study supports ongoing quality efforts to shorten doorto-procedure times worldwide. The American Heart/ American Stroke Association Target: Stroke III program ${ }^{26}$ recommends 12 specific best practice strategies to accelerate door-to-device times, including prearrival notification of the neurointerventional team, rapid availability around the clock of the neurointerventional team, transfer directly from the brain imaging suite to the angiography suite, prereadied EVT equipment sets, rapid anesthesia protocols, and prompt workflow data feedback after every case. A particularly notable finding from the current investigation is the similarity of door-tointervention delays in worsening outcome between patients treated late ( $>4-12$ hours) and patients treated early ( $\leq 4$ hours) after last known well time. Among all patients who qualify for EVT based on imaging, regardless of time within the first 12 hours from onset, speedy postarrival workflow is necessary to maximize patient benefit. ${ }^{27}$

\section{Limitations}

This study has limitations. The patient data come from randomized clinical trials with strict inclusion criteria that excluded patients with preexisting disability. Therefore, the outcomes of this analysis may not accurately reflect the changes of DALYs associated with EVT when applied to broader populations in routine practice, but they are fully applicable to patients who meet the highest level of guideline-endorsed eligibility for treatment, as these criteria reflect the clinical trial populations. ${ }^{1}$ In addition, the analyzed trials had heterogeneous entry criteria and imaging methods. However, all pa- 


\begin{tabular}{|c|c|c|c|c|}
\hline \multirow[b]{2}{*}{ Variable } & \multicolumn{4}{|c|}{ Amount of healthy life-time lost } \\
\hline & Hours & Days & Months & Years \\
\hline \multicolumn{5}{|c|}{ Amount of delay in DTP time interval } \\
\hline $1 \mathrm{~s}$ & 2.2 & 0.09 & 0.003 & 0.00026 \\
\hline $1 \mathrm{~min}$ & 134.0 & 5.6 & 0.18 & 0.015 \\
\hline $10 \mathrm{~min}$ & 1340.3 & 55.8 & 1.8 & 0.15 \\
\hline $1 \mathrm{~h}$ & 8041.7 & 335.1 & 11.0 & 0.92 \\
\hline \multicolumn{5}{|c|}{ Amount of delay in DTR time interval } \\
\hline $1 \mathrm{~s}$ & 2.4 & 0.10 & 0.003 & 0.00028 \\
\hline $1 \mathrm{~min}$ & 146.4 & 6.2 & 0.20 & 0.017 \\
\hline $10 \mathrm{~min}$ & 1464.4 & 61.0 & 2.0 & 0.17 \\
\hline $1 \mathrm{~h}$ & 8786.3 & 366.1 & 12.0 & 1.00 \\
\hline
\end{tabular}

Abbreviations:

DTP, door-to-puncture; DTR, door-to-reperfusion.

a The early-treated population was defined as onset to puncture $\leq 4$ hours. tients had disabling strokes with LVO and limited extent of early ischemic changes on baseline imaging. The utility scores used here were not directly collected from patients in each trial. Therefore, the DALYs lost may vary when applied to different populations. However, the utility weights were derived from 2 studies, 1 of a broad stroke patient population ${ }^{19}$ and the other of international stroke health professionals ${ }^{2 \mathrm{O}}$; the similar weights identified in these studies with contrasting methods suggest a broadly applicable weighting. This study analyzed mostly patients enrolled in trials of relatively early EVT with limited imaging selection. Findings are not directly applicable to patients treated in late time windows up to 24 hours after onset after sophisticated imaging selection. ${ }^{28,29}$ Finally, we projected lifetime disability status based on 90-day outcomes. Up to $25 \%$ of stroke patients may experience improvement in mRS scores between
3 months and 1 year after stroke. ${ }^{30}$ This finding indicates that our estimates of the magnitude of DALYs lost with care process delays are likely conservative, as some patients will have lateoutcome time periods characterized by reduced disability.

\section{Conclusions}

Results of this study suggest that timely successful reperfusion is an important factor associated with EVT outcome. Delays, particularly after arrival to the hospital until reperfusion is achieved, may result in substantial losses in years of healthy life for patients. Efforts to optimize workflow and eliminate barriers preventing timely patient evaluation and treatment within health care systems are warranted.

\section{ARTICLE INFORMATION}

Accepted for Publication: March 5, 2021.

Published Online: May 3, 2021.

doi:10.1001/jamaneurol.2021.1055

Author Affiliations: Calgary Stroke Program, Hotchkiss Brain Institute, Cumming School of Medicine, University of Calgary, Calgary, Alberta, Canada (Almekhlafi, Goyal, Demchuk, Hill); Department of Clinical Neurosciences, University of Calgary, Calgary, Alberta, Canada (Almekhlafi, Goyal, Hill); Department of Radiology, University of Calgary, Calgary, Alberta, Canada (Almekhlafi, Goyal, Demchuk, Hill); Department of Community Health Sciences, University of Calgary, Calgary, Alberta, Canada (Almekhlafi, Goyal); Erasmus MC University Medical Center Depts of Neurology, Rotterdam, the Netherlands (Dippel); Department of Radiology and Nuclear Medicine, Amsterdam University Medical Centers, Amsterdam, the Netherlands (Majoie); Department of Medicine and Neurology, Melbourne Brain Centre at the Royal Melbourne Hospital, University of Melbourne, Parkville, Victoria, Australia (Campbell); Institute of Neuroscience \& Psychology, University of Glasgow, Glasgow, Scotland, United Kingdom (Muir); Department of Diagnostic and Interventional Neuroradiology, Université de Lorraine, Inserm, IADI, CHRU Nancy, Nancy, France (Bracard); CHRU-Nancy, INSERM, Université de Lorraine, CIC Clinical Epidemiology, Nancy, France (Guillemin); Department of Neurology, Cooper
University Health Care, Camden, New Jersey (Jovin); Department of Radiology, Royal Melbourne Hospital, University of Melbourne, Parkville, Victoria, Australia (Mitchell); Translational and Clinical Research Institute, Newcastle University, Newcastle upon Tyne, United Kingdom (White); Department of Medicine, University of Calgary, Calgary, Alberta, Canada (Hill); Altair Biostatistics, St Louis Park, Minnesota (Brown); Department of Neurology, David Geffen School of Medicine, University of California, Los Angeles (Saver).

Author Contributions: Drs Almekhlafi and Saver had full access to all of the data in the study and take responsibility for the integrity of the data and the accuracy of the data analysis. Concept and design: Almekhlafi, Goyal, Jovin, Mitchell, Hill, Saver.

Acquisition, analysis, or interpretation of data: Almekhlafi, Goyal, Dippel, Majoie, Campbell, Muir, Demchuk, Bracard, Guillemin, White, Hill, Brown, Saver.

Drafting of the manuscript: Almekhlafi, Brown. Critical revision of the manuscript for important intellectual content: Goyal, Dippel, Majoie, Campbell, Muir, Demchuk, Bracard, Guillemin, Jovin, Mitchell, White, Hill, Saver.

Statistical analysis: Almekhlafi, Brown, Saver. Obtained funding: Goyal, Campbell, Demchuk, Hill. Administrative, technical, or material support: Demchuk, Mitchell, White.

Supervision: Goyal, Demchuk, White, Saver.
Conflict of Interest Disclosures: Dr Almekhlafi reported being a member of the scientific advisory board of Palmera Medical, Inc. Dr Goyal reported receiving grants from Medtronic during the conduct of the study, personal fees from Mentice Consulting on acute stroke workflow, personal fees from Medtronic Consulting on acute stroke intervention, personal fees from Microvention for advice on acute stroke intervention, and personal fees from Stryker for advice on acute stroke products outside the submitted work. Dr Dippel reported receiving grants from Dutch Heart Foundation, grants from Brain Foundation Netherlands, grants from The Netherlands Organisation for Health Research and Development, grants from Health Holland Top Sector Life Sciences \& Health all paid to institution, grants from AngioCare BV, grants from Covidien/ EV3, grants from MEDAC Gmbh/LAMEPRO, grants from Penumbra Inc, grants from Top Medical/ Concentric, grants from Stryker, grants from Stryker European Operations BV, grants from Medtronic, and grants from Thrombolytic Science, LLC, all unrestricted, paid to institution outside the submitted work. Dr Majoie reported grants from CVON/Dutch Heart Foundation paid to institution, grants from European Commission paid to institution, grants from Dutch Health Evaluation Program paid to institution, grants from Stryker paid to institution, and grants from TWIN Foundation paid to institution outside the submitted work; and being a shareholder of Nico-lab. Dr Muir reported receiving nonfinancial 
support from Boehringer Ingelheim, personal fees from Biogen, personal fees from Bayer, personal fees from AbbVie, personal fees from ReNeuron, and personal fees from Daiichi Sankyo outside the submitted work. Dr Demchuk reported receiving an unrestricted grant from Medtronic for the ESCAPE trial and honoraria from Medtronic for CME events during the conduct of the study and Circle NVI stock ownership outside the submitted work; in addition, Dr Demchuk received a patent for Circle NVl-issued stroke imaging software. Dr Jovin reported being an investor/advisor for Anaconda. Route92, Methinks, Viz.ai, and FreeOx, other support from Corindus Advisor, personal fees from Contego for serving on a medical screening committee, personal fees from Cerenovus for serving on a data safety and monitoring board and a steering committee, grants from Stryker for AURORA, DAWN, grants from Medtronic for REACT, and nonfinancial support from Fundacio Ictus for RACECAT/REVASCAT-related travel outside the submitted work. Dr Mitchell reported receiving institutional research support from Stryker and institutional research support from Medtronic outside the submitted work. Dr White reported an unrestricted educational grant to institution from Stryker, an unrestricted educational grant to institution from Penumbra, an unrestricted educational grant to institution from Medtronic, grants from Microvention, and personal fees from Microvention outside the submitted work. Dr Hill reported a grant from Medtronic LLC to the University of Calgary for the HERMES Collaboration during the conduct of the study; a grant from NoNO Inc to the University of Calgary, a grant from Boehringer Ingelheim Canada to the University of Calgary, and a grant from Biogen Inc to the University of Calgary outside the submitted work; in addition, Dr Hill received a patent for US Patent Office Number 62/086,077 licensed to Circle NVI; and is a director of the Canadian Federation of Neurological Sciences, a not-for-profit group, is a director of the Canadian Stroke Consortium, a not-for-profit group, is a director of Circle NeuroVascular Inc., and has received grant support from Alberta Innovates Health Solutions, CIHR, Heart \& Stroke Foundation of Canada, and National Institutes of Neurological Disorders and Stroke. Dr Brown reported receiving personal fees from University of Calgary HERMES for statistical consulting during the conduct of the study; personal fees from Medtronic for statistical consulting outside the submitted work. Dr Saver reported receiving personal fees from Medtronic for contracted hourly payments and travel reimbursement for service on trial steering committee(s), making recommendations regarding rigorous trial design and conduct, personal fees from Stryker for contracted hourly payments and travel reimbursement for service on trial steering committee(s), making recommendations regarding rigorous trial design and conduct, personal fees from Cerenovus for contracted hourly payments and travel reimbursement for service on trial steering committee(s), making recommendations regarding rigorous trial design and conduct, and personal fees from Rapid Medical for contracted stock options and travel reimbursement for clinical trial steering committee service, advising on rigorous trial design and conduct outside the submitted work; and The University of California Regents have patent rights in retrieval devices. No other disclosures were reported.
Funding/Support: Medtronic provided an unrestricted grant to the University of Calgary HERMES Coordinating Center for this study.

Role of the Funder/Sponsor: Medtronic had no role in the design and conduct of the study; collection, management, analysis, and interpretation of the data; preparation, review, or approval of the manuscript; and decision to submit the manuscript for publication.

Group Information: The members of the HERMES Trialists Collaboration are listed in the Supplement.

\section{REFERENCES}

1. Powers WJ, Rabinstein AA, Ackerson T, et al. Guidelines for the early management of patients with acute ischemic stroke: 2019 update to the 2018 guidelines for the early management of acute ischemic stroke: a guideline for healthcare professionals from the American Heart Association/American Stroke Association. Stroke. 2019;50(12):e344-e418. doi:10.1161/STR. 0000000000000211

2. Goyal M, Menon BK, van Zwam WH, et al; HERMES Collaborators. Endovascular thrombectomy after large-vessel ischaemic stroke: a meta-analysis of individual patient data from five randomised trials. Lancet. 2016;387(10029):17231731. doi:10.1016/S0140-6736(16)00163-X

3. Saver JL, Fonarow GC, Smith EE, et al. Time to treatment with intravenous tissue plasminogen activator and outcome from acute ischemic stroke. JAMA. 2013;309(23):2480-2488. doi:10.1001/jama. 2013.6959

4. Jahan R, Saver JL, Schwamm LH, et al. Association between time to treatment with endovascular reperfusion therapy and outcomes in patients with acute ischemic stroke treated in clinical practice. JAMA. 2019;322(3):252-263. doi:10.1001/jama.2019.8286

5. Saver JL, Goyal M, van der Lugt A, et al; HERMES Collaborators. Time to treatment with endovascular thrombectomy and outcomes from ischemic stroke: a meta-analysis. JAMA. 2016;316 (12):1279-1288. doi:10.1001/jama.2016.13647

6. Kunz WG, Hunink MG, Almekhlafi MA, et al; HERMES Collaborators. Public health and cost consequences of time delays to thrombectomy for acute ischemic stroke. Neurology. 2020;95(18): e2465-e2475. doi:10.1212/WNL. 0000000000010867

7. Berkhemer OA, Fransen PS, Beumer D, et al; MR CLEAN Investigators. A randomized trial of intraarterial treatment for acute ischemic stroke. N Engl J Med. 2015;372(1):11-20. doi:10.1056/ NEJMoa1411587

8. Goyal M, Demchuk AM, Menon BK, et al; ESCAPE Trial Investigators. Randomized assessment of rapid endovascular treatment of ischemic stroke. N Engl J Med. 2015;372(11):10191030. doi:10.1056/NEJMoa1414905

\section{Campbell BC, Mitchell PJ, Kleinig TJ, et al;} EXTEND-IA Investigators. Endovascular therapy for ischemic stroke with perfusion-imaging selection. N Engl J Med. 2015;372(11):1009-1018. doi:10.1056/ NEJMoa1414792

10. Saver JL, Goyal M, Bonafe A, et al; SWIFT PRIME Investigators. Stent-retriever thrombectomy after intravenous t-PA vs t-PA alone in stroke.
N Engl J Med. 2015;372(24):2285-2295. doi:10.1056/NEJMoa1415061

11. Jovin TG, Chamorro A, Cobo E, et al; REVASCAT Trial Investigators. Thrombectomy within 8 hours after symptom onset in ischemic stroke. N Engl J Med. 2015;372(24):2296-2306. doi:10.1056/NEJMoa1503780

12. Muir KW, Ford GA, Messow C-M, et al; PISTE Investigators. Endovascular therapy for acute ischaemic stroke: the Pragmatic Ischaemic Stroke Thrombectomy Evaluation (PISTE) randomised, controlled trial. J Neurol Neurosurg Psychiatry. 2017;88(1):38-44. doi:10.1136/jnnp-2016314117

13. Bracard S, Ducroca $X$, Mas JL, et al; THRACE Investigators. Mechanical thrombectomy after intravenous alteplase versus alteplase alone after stroke (THRACE): a randomised controlled trial. Lancet Neurol. 2016;15(11):1138-1147. doi:10.1016/S1474-4422(16)30177-6

14. Campbell BCV, van Zwam WH, Goyal M, et al; HERMES Collaborators. Effect of general anaesthesia on functional outcome in patients with anterior circulation ischaemic stroke having endovascular thrombectomy versus standard care: a meta-analysis of individual patient data. Lancet Neurol. 2018;17(1):47-53. doi:10.1016/S1474-4422(17) 30407-6

15. Murray CJL, Lopez AD; World Health Organization; World Bank \& Harvard School of Public Health. The global burden of disease: a comprehensive assessment of mortality and disability from diseases, injuries, and risk factors in 1990 and projected to 2020: summary. Published 1996. Accessed March 29, 2021. https://apps. who.int/iris/handle/10665/41864

16. Slot KB, Berge E, Dorman P, Lewis S, Dennis M, Sandercock P; Oxfordshire Community Stroke Project, the International Stroke Trial (UK); Lothian Stroke Register. Impact of functional status at six months on long term survival in patients with ischaemic stroke: prospective cohort studies. BMJ. 2008;336(7640):376-379. doi:10.1136/bmj.39456. 688333.BE

17. Eriksson M, Norrving $B$, Terént A, Stegmayr $B$. Functional outcome 3 months after stroke predicts long-term survival. Cerebrovasc Dis. 2008;25(5): 423-429. doi:10.1159/000121343

18. Hong K-S, Saver JL. Years of disability-adjusted life gained as a result of thrombolytic therapy for acute ischemic stroke. Stroke. 2010;41(3):471-477. doi:10.1161/STROKEAHA.109.571083

19. Rivero-Arias $O$, Ouellet M, Gray A, Wolstenholme J, Rothwell PM, Luengo-Fernandez R. Mapping the modified Rankin scale (mRS) measurement into the generic EuroQol (EQ-5D) health outcome. Med Decis Making. 2010;30(3):341354. doi:10.1177/0272989X09349961

20. Hong K-S, Saver JL. Quantifying the value of stroke disability outcomes: WHO global burden of disease project disability weights for each level of the modified Rankin Scale. Stroke. 2009;40(12): 3828-3833. doi:10.1161/STROKEAHA.109.561365

21. Chaisinanunkul N, Adeoye O, Lewis RJ, et al; DAWN Trial and MOST Trial Steering Committees; Additional contributors from DAWN Trial Steering Committee. Adopting a patient-centered approach to primary outcome analysis of acute stroke trials using a utility-weighted modified Rankin scale. Stroke. 
2015;46(8):2238-2243. doi:10.1161/STROKEAHA.114. 008547

22. Meretoja A, Keshtkaran M, Tatlisumak T, Donnan GA, Churilov L. Endovascular therapy for ischemic stroke: save a minute-save a week. Neurology. 2017;88(22):2123-2127. doi:10.1212/WNL. 0000000000003981

23. Menon BK, Sajobi TT, Zhang Y, et al. Analysis of workflow and time to treatment on thrombectomy outcome in the endovascular treatment for small core and proximal occlusion ischemic stroke (ESCAPE) randomized, controlled trial. Circulation. 2016;133(23):2279-2286. doi:10.1161/ CIRCULATIONAHA.115.019983

24. Ribo M, Molina CA, Cobo E, et al; REVASCAT Trial Investigators. Association between time to reperfusion and outcome is primarily driven by the time from imaging to reperfusion. Stroke. 2016;47 (4):999-1004. doi:10.1161/STROKEAHA.115.011721

25. Fiehler J. The time-reset effect. Clin Neuroradiol. 2017;27(1):3-5. doi:10.1007/s00062-017-0561-4

26. Target: Stroke Phase III Guide. American Heart/American Stroke Association. Accessed February 2021. https://www.heart.org/-/media/ files/professional/quality-improvement/targetstroke/target-stroke-phase-iii/9-17-update/finaldoor-to-device-best-practice-one-pager.pdf?la=en

27. Goyal M, Almekhlafi M, Dippel DW, et al; HERMES Collaborators. Rapid alteplase administration improves functional outcomes in patients with stroke due to large vessel occlusions: meta-analysis of the noninterventional arm from the HERMES collaboration. Stroke. 2019;50(3): 645-651. doi:10.1161/STROKEAHA.118.021840
28. Albers GW, Marks MP, Kemp S, et al; DEFUSE 3 Investigators. Thrombectomy for stroke at 6 to 16 hours with selection by perfusion imaging. N Engl J Med. 2018;378(8):708-718. doi:10.1056/ NEJMoa1713973

29. Nogueira RG, Jadhav AP, Haussen DC, et al; DAWN Trial Investigators. Thrombectomy 6 to 24 hours after stroke with a mismatch between deficit and infarct. N Engl J Med. 2018;378(1):11-21. doi:10. 1056/NEJMoa1706442

30. Ganesh A, Luengo-Fernandez R, Rothwell PM. Late functional improvement and 5-year poststroke outcomes: a population-based cohort study. J Neurol Neurosurg Psychiatry. 2020;91(8):831-839. doi:10.1136/jnnp-2019-322365 